\title{
Modelling the compaction of plastic particle packings
}

\author{
Saeid Nezamabadi ${ }^{1,2,3}$ (ID Mojtaba Ghadiri ${ }^{3} \cdot$ Jean-Yves Delenne $^{2} \cdot$ Farhang Radjai $^{1}$
}

\begin{abstract}
Soft particle materials such as some pharmaceutical and food products are composed of particles that can undergo large deformations under low confining pressures without rupture. The rheological and textural properties of these materials are thus governed by both particle rearrangements and particle shape changes. For the simulation of soft particle materials, we present a numerical technique based on the material point method, allowing for large elasto-plastic particle deformations. Coupling the latter with the contact dynamics method makes it possible to deal with contact interactions between particles. We investigate the compaction of assemblies of elastic and plastic particles. For plastic deformations, it is observed that the applied stress needed to achieve high packing fraction is lower when plastic hardening is small. Moreover, predictive models, relating stress and packing fraction, are proposed for the compaction of elastic and plastic particles. These models fit well our simulation results. Furthermore, it is found that the evolution of the coordination number follows a power law as a function of the packing fraction beyond jamming point of hard particle packings.
\end{abstract}

Keywords Material point method · Contact dynamics · Granular materials · Powders · Plastic particles

\section{Introduction}

Compaction is one of the most important manufacturing processes of particulate materials, used in a number of industries, including food, pharmaceutical, chemical, powder metallurgy, ceramic and nuclear industries. This process transforms loose particulate materials into a compacted product with a higher packing fraction $[1,2]$. During the initial stage of compaction, particles can rearrange while keeping their initial shapes. Advancing in compaction process, particles undergo large (elastic and/or plastic) deformations, which cause particle shape changes. The latter allows achieving high packing fractions, which considerably exceed the random close packing $(\mathrm{RCP})$ limit.

In order to understand the compaction process better, it can be modelled using two main numerical techniques: (i) continuum approach, in which particulate materials are considered

Saeid Nezamabadi

saeid.nezamabadi@umontpellier.fr

1 LMGC, CNRS, University of Montpellier, Montpellier, France

2 IATE, INRAE, Montpellier SupAgro, University of Montpellier, Montpellier, France

3 School of Chemical and Process Engineering, University of Leeds, Leeds LS2 9JT, UK as a continuum medium [3-5]; and (ii) discrete element modelling, in which the behaviour and interaction of individual particles are taken into account [6,7]. The first method is based on defining macroscopic constitutive laws taking account of micro-dynamic information of particles. The discrete element method (DEM) is generally used in the context of the second approach. In DEM, particles are assumed to be slightly deformable through different contact theories such as the Hertz contact theory, which is only valid up to about $10 \%$ of strain. However, in the context of compaction process modelling, this assumption is too crude. For realistic simulation of compaction, it is hence primordial to combine a continuum representation of the particles, allowing for their deformation according to a prescribed constitutive model, with appropriate contact interactions between particles [8,9].

In this paper, our numerical approach is based on a parallel implicit material point method (MPM) in association with the contact dynamics (CD) method for the treatment of frictional contacts between particles [9-11]. Using this approach, we first study the behaviour of a single plastic particle subjected to axial strain. This method is then applied to the compaction simulation of a packing of plastic particles. The packing can reach high packing fractions beyond the "jamming" point by particle shape changes and still deform plastically. As we 
shall see, the particle plastic behaviour affects the stress level and its evolution during compaction.

In the following, we first briefly introduce the numerical approaches in Sect. 2. Then, Sect. 3 is devoted to the behaviour of a single particle subjected to diametrical compression. We focus in Sect. 4 on the compaction process of packings of plastic particles. We conclude with a brief summary of this work.

\section{Methodology}

In this section, our methodology based on the coupling of an implicit MPM formulation and the contact dynamics method is described briefly. This approach was introduced in the previous works [9-11]. Here, we present its extension to plastic behaviour in the context of the infinitesimal strain theory.

We consider a domain $\Omega$ in $\mathbb{R}^{D}, D$ being the domain dimension, describing a continuum body. Its conservation of mass is described by the following continuity equation:

$$
\frac{\partial \rho(\mathbf{x}, t)}{\partial t}+\nabla(\rho(\mathbf{x}, t) \cdot \mathbf{v}(\mathbf{x}, t))=0 \quad \text { in } \Omega
$$

and the Cauchy form of conservation of linear momentum for this body is given by the equation:

$$
\nabla \cdot \sigma(\mathbf{x}, t)+\mathbf{b}(\mathbf{x}, t)=\rho(\mathbf{x}, t) \mathbf{a}(\mathbf{x}, t) \quad \text { in } \Omega .
$$

In the above equations, $\rho(\mathbf{x}, t), \mathbf{v}(\mathbf{x}, t), \sigma(\mathbf{x}, t), \mathbf{b}(\mathbf{x}, t)$ and $\mathbf{a}(\mathbf{x}, t)$ represent, respectively, material density, velocity, Cauchy stress tensor, body force and acceleration at position $\mathbf{x}$ and time $t$.

The momentum relation (2) must be complemented with a constitutive relationship, which relates the Cauchy stress, $\boldsymbol{\sigma}$, to the strain tensor, $\boldsymbol{\epsilon}\left(\boldsymbol{\epsilon}=\frac{1}{2}\left(\nabla \mathbf{u}+{ }^{t} \nabla \mathbf{u}\right)\right.$, $\mathbf{u}$ being the displacement field). Here, we consider a linear isotropic elastic material model and a rate-independent elasto-plastic model based on the bilinear isotropic hardening [12]. The latter uses the von Mises yield criteria coupled with an isotropic work hardening assumption. It is called bilinear because just two lines define the stress-strain curve with a transition point defined as the yield stress $\sigma_{y}$ : one to describe the linear elastic region with Young's modulus, $E$, and another to the plastic with hardening modulus, $H$.

In MPM, the continuum body is discretised by a set of material points with fixed mass. According to the assumption of the fixed material point mass, the mass conservation relation (1) is automatically satisfied. The MPM can be considered as a finite element method (FEM) with moving integration points (material points) that serve to compute the FEM integrals. The MPM discretises these integrals through a Dirac delta function. Hence, the weak form of the momen- tum relation (2) in its discretised version can be written in the presence of contact interactions between several bodies, in the following form:

$$
\mathbf{M} \mathbf{a}_{\text {node }}(t)=\mathbf{f}^{\text {int }}(t)+\mathbf{f}^{\text {ext }}(t)+\mathbf{f}^{\mathrm{C}}(t)
$$

where $\mathbf{a}_{\text {node }}$ is the nodal acceleration, $\mathbf{f}^{\mathrm{C}}$ represents the nodal contact force (see below), and

$$
\begin{aligned}
& \mathbf{M}=\sum_{p=1}^{N_{p}} m_{p} \mathbf{N}_{p} \quad \text { Lumped mass matrix } \\
& \mathbf{f}^{\mathrm{int}}=-\sum_{p=1}^{N_{p}} \mathbf{G}_{p} \boldsymbol{\sigma}_{p} V_{p} \quad \text { Internal force vector, } \\
& \mathbf{f}^{\text {ext }}=\sum_{p=1}^{N_{p}} \mathbf{N}_{p} \mathbf{b}_{p}+\mathbf{f}^{\mathbf{S}} \quad \text { Sum of body forces and } \\
& \text { surface tractions, } \mathbf{f}^{\mathrm{S}} \text {. }
\end{aligned}
$$

In the above relations, $m_{p}$ and $V_{p}$ are the mass and volume of a material point $p$, and $N_{p}$ indicates the number of material points. $\mathbf{N}_{p}$ is the interpolation matrix (or the shape function matrix) at the material point $p$. It relates the quantities associated with the material points (displacement, position, etc.) to nodal variables of the element to which the material point belongs. $\mathbf{G}_{p}$ is the gradient of the shape function $\mathbf{N}_{p}$.

Equation (3) is solved using an implicit MPM approach described in the previous work [10]. The nodal solutions are then projected onto the material points, allowing for updating the information carried by these points. Note that the implementation of the plastic constitutive law in the implicit MPM approach is similar to the finite element method since the MPM can be considered as a minor modification of the classical FEM. It is also worth noting that here, the evolution of the material point volume $V_{p}$ is considered to change from time $t$ to $t+\Delta t$ as

$$
V_{p}(t+\Delta t)=\operatorname{det}\left(\mathbf{I}+\nabla \mathbf{u}_{p}{ }_{t}^{t+\Delta t}\right) V_{p}(t)
$$

where $\mathbf{u}_{p}{ }_{t}^{t+\Delta t}$ represents the displacement filed of the material point $p$ between the times $t$ and $t+\Delta t$ and $\mathbf{I}$ is the second-order identity tensor. This evolution is based on the finite strain MPM formulation as defined by [11].

Finally, since our MPM formulation is used to deal with the soft particle systems, the nodal contact forces $\mathbf{f}^{\mathrm{C}}$ between deformable particles are computed using a multi-mesh contact algorithm coupling with the contacts dynamics method. In the context of multi-mesh algorithm, each soft particle maps in its proper background mesh. A contact point at the interface between two particles may be treated by introducing a common background mesh with the same type of grids for the transfer of nodal quantities from proper meshes to 


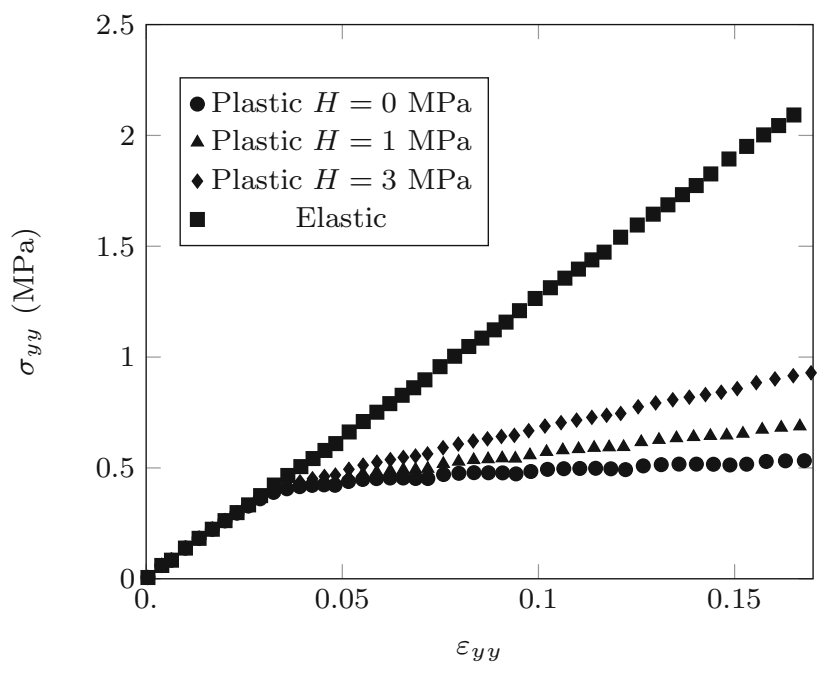

Fig. 1 Stress-strain diagrams for the single elastic and elasto-plastic (with several values of hardening) particles subjected to diametrical compression

the common mesh. Considering an implicit MPM procedure, the CD method, which is based on an implicit time-stepping scheme, is a natural choice for the treatment of contact points. For more details about the presented contact algorithm, see [9-11].

\section{Diametrical compression of a single cylindrical particle}

Let us first consider the diametrical compression of a single soft cylindrical particle. Its diameter is $D=60 \mathrm{~mm}$ and is compacted between two rigid walls. The top wall is moved down with a constant velocity of $0.5 \mathrm{~m} / \mathrm{s}$, while the bottom wall is fixed. We performed the two-dimensional MPM simulation in plane strain conditions to model this problem with a time step of $\Delta t=0.1 \mu \mathrm{s}$. The computation domain was meshed with four-node quadrangular elements, and an initial distribution of four material points per element was used. A cubic spline interpolation is also used for each individual material point and maps the material point values to nodes a maximum distance of two grid nodes away in either direction [13].

As mentioned above, two types of material deformation models are considered: elastic and elasto-plastic with linear isotropic hardening. For the two deformation models, Young's modulus, Poisson's ratio and density of the particle are set to $E=10 \mathrm{MPa}, v=0.45$ and $\rho=1000 \mathrm{~kg} / \mathrm{m}^{3}$, respectively. For elasto-plastic one, the yield stress is equal to $\sigma_{y}=0.4 \mathrm{MPa}$ and we consider three values of hardening $H=0,1$ and $3 \mathrm{MPa}$. Figure 1 shows the Cauchy stress $\sigma_{y y}$ as a function of the strain $\varepsilon_{y y}$. We observe a linear behaviour for the elastic particle though the particle has a cylindrical shape.
It may be explained by the small imposed deformation; i.e. $\varepsilon<0.17$. For the elasto-plastic particles, a linear response until $\sigma_{y y} \simeq 0.4 \mathrm{MPa}$, which corresponds to the yield stress $\sigma_{y}$ and a linear plastic behaviour beyond. The slope of the plastic parts increases as hardening augments.

\section{Uniaxial compression of an assembly of cylindrical shape particles}

In this section, the MPM simulations are carried out to investigate the compaction of an assembly of elasto-plastic soft cylindrical particles. The assembly contains 300 discs confined inside a rectangular box in which only the top wall is mobile and moves down with a constant velocity of $2 \mathrm{~m} / \mathrm{s}$. It allows the uniaxial compression of the assembly. Its initial configuration is prepared using DEM simulations. To avoid long-range ordering, a uniform distribution of the particles by volume fractions in the range $[2,4] \mathrm{mm}$ is introduced. The same material responses as for the single particle analysis, reported above, are considered. In the MPM simulations, the time step is set to be $\Delta t=0.1 \mu \mathrm{s}$ and no friction is introduced. The gravitational acceleration is also set to be zero to avoid stress gradients.

Figure 2 shows initial configuration and snapshots of the compact state of the assembly for the elastic and elastoplastic behaviours. Due to particle shape changes, the whole space is nearly filled at the end of the compaction process, and so, the packing fraction $\left(\Phi=V_{S} / V\right.$, where $V_{S}$ is the volume of particles and $V$ the total volume) increases. We observe that the shapes of the particles change gradually from circular to polygonal and the plastic particles at the end have a polygonal shape with larger curvatures in comparison with the elastic particles; see the zoom on the deformed packings in Fig. 2. Indeed, the plasticity allows these particles overfill the pores for much lower stress than the elastic ones.

In order to highlight the above feature, the mean value of maximum curvature of the particles $\kappa$ and the packing fraction $\Phi$ as a function of the applied stress $\sigma$ is presented in Fig. 3. $\sigma$ is computed from the contact forces acting on the bottom wall. As mentioned above, the plastic particles achieve higher values of $\kappa$ by smaller applied stress $\sigma$ than the elastic particles. We also observe that for the plastic particles, when the hardening $H$ decreases, the curvature $\kappa$ increases; i.e. one needs less applied stress to fill the pores of a particulate system under compression when the hardening of plastic particles is small. This point also features in the evolution of the packing fraction $\Phi$ as a function of the applied stress $\sigma$. A nonlinear behaviour for the elastic and plastic particles with different rates is observed. To compress the elastic particle packing, we require a larger force than for the plastic particles, and the required force increases for the plastic particles when the hardening augments. 


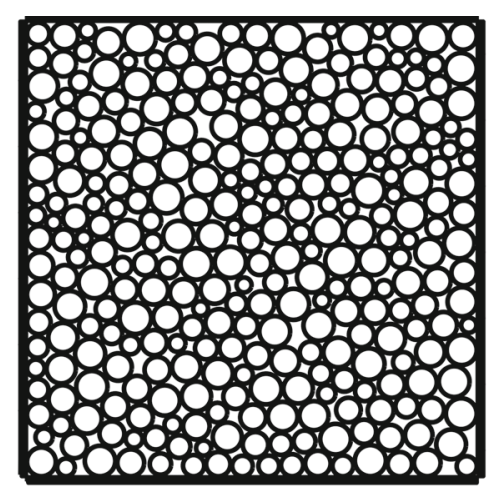

(a) Initial state: $\Phi=0.78$
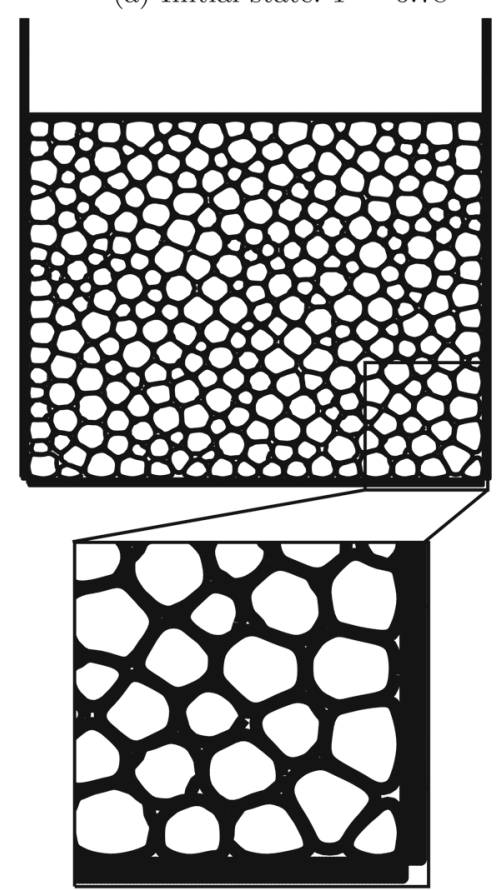

(c) Plastic $H=3 \mathrm{MPa}$

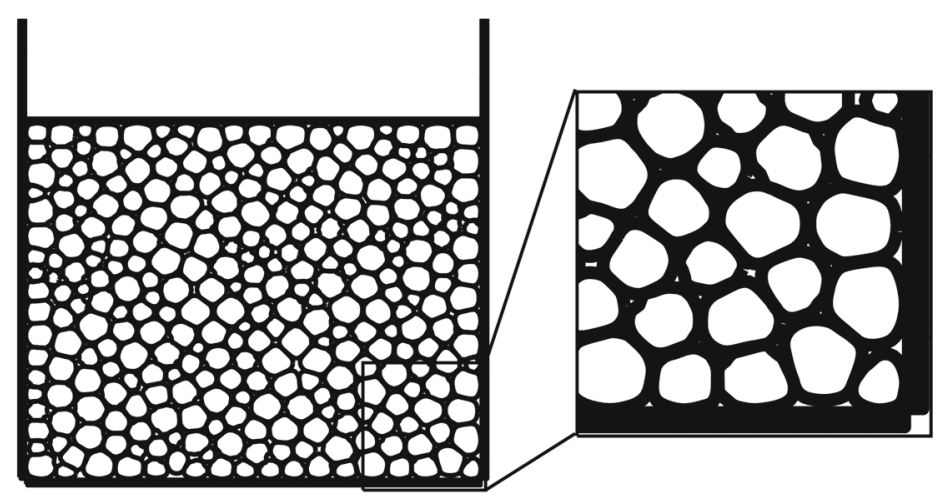

(b) Elastic
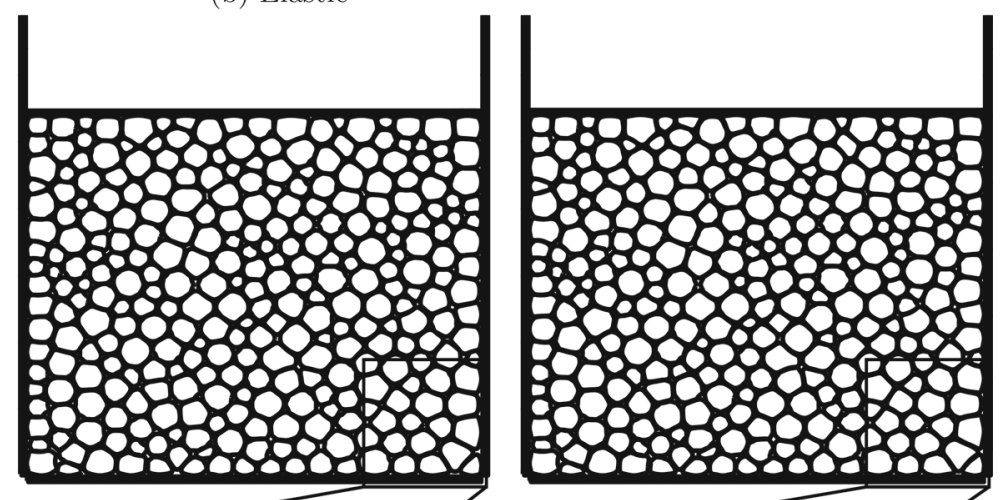

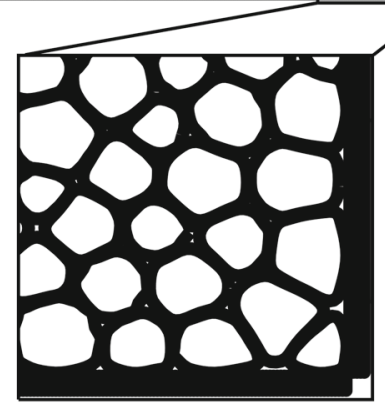

(d) Plastic $H=1 \mathrm{MPa}$

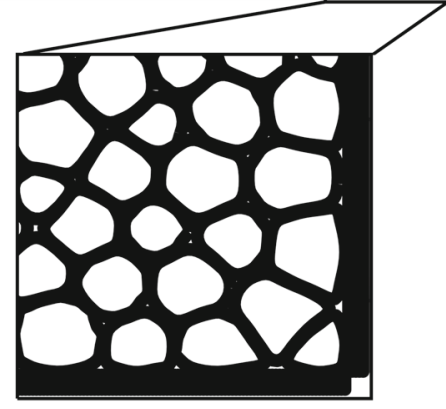

(e) Plastic $H=0 \mathrm{MPa}$

Fig. 2 A snapshot of the initial configuration (a); snapshots of the compacted packings of particles with elastic and elasto-plastic behaviours (with three values of the hardening parameter $H$ ) at packing fraction of $\Phi=0.97$, and zoom on the particles inside a bottom right window of the deformed packings $(\mathbf{b}-\mathbf{e})$

In Fig. 3, we also observe that the plastic results diverge from the elastic ones for $\sigma \gtrsim 0.35 \mathrm{MPa}$. This value is smaller than the yield stress of one plastic particle $\left(\sigma_{y}=0.4 \mathrm{MPa}\right)$ since not all the particles in the packing undergo plastic deformation at the same time. Moreover, for all cases, a regime change is observed around the packing fraction of 0.8 . This change point can be considered as the jamming point, i.e. $\Phi_{c} \simeq 0.8$. Above this point, particle rearrangement is negligible and the particle shape change controls essentially the packing evolution. One can hence assume that the packing behaviours beyond the jamming point are almost like a continuum medium. Considering the $\mathrm{P}$-wave modulus definition, this assumption allows obtaining the applied stress $\sigma$ as a function of the cumulative vertical strain $\varepsilon\left(\varepsilon=\ln \left(h / h_{i}\right)\right.$, with $h$ as the current height of the packing and $h_{i}$ its initial height) for the elastic particles:

$\sigma-\sigma_{c}=M^{\text {eff }}\left(\varepsilon-\varepsilon_{c}\right)$

where $\sigma_{c}$ and $\varepsilon_{c}$ are, respectively, the applied stress and the vertical strain at the jamming point, and $M^{\text {eff }}$ denotes the effective elastic P-wave modulus to be defined. Based on Eq. (5), one may relate $\sigma$ to the packing fraction $\Phi$ and the mean coordination number $Z$ for the elastic particles (see "Appendix 1"):

$\sigma=-\frac{M^{\text {eff }}}{1+\frac{M^{\mathrm{eff}}}{c_{1} K Z \Phi}}\left(\ln (\Phi)+c_{2}\right)$ 


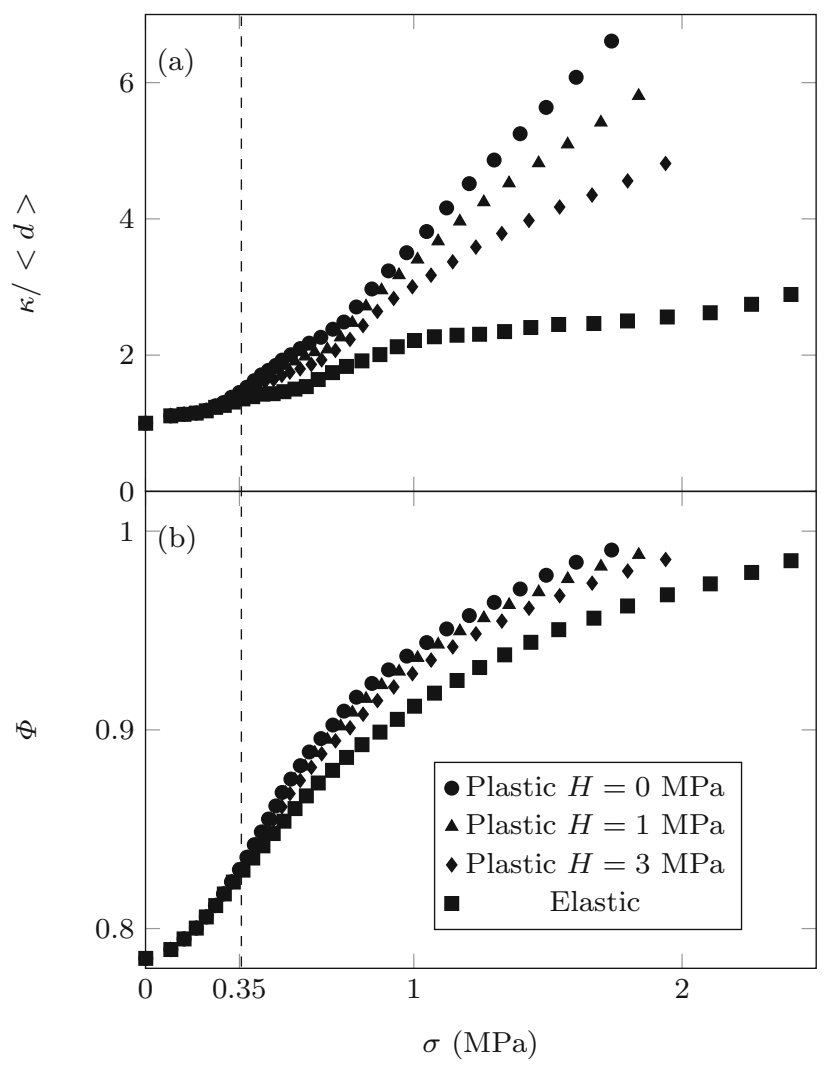

Fig. 3 Mean value of maximum curvature $\kappa$ of the particles normalised by their mean diameter $\langle d>$ (a) and the packing fraction $\Phi(\mathbf{b})$ as a function of the applied stress $\sigma$ for elastic and elasto-plastic material behaviours

where $K$ denotes the particle bulk modulus $(K=\lambda+\mu$ in 2D, with $\lambda$ and $\mu$ as Lamé parameters), $c_{1}$ represents a constant parameter depending on the particle material behaviour and $c_{2}$ is a constant term. Note that since $Z$ can also be defined as a function of $\Phi$ (see Eq. (17)), $\sigma$ is only related to the packing fraction $\Phi$ via Eq. (6).

In our previous work [11], considering the Voigt rule of mixtures gives $M^{\text {eff }}=\Phi M$ with $M$ as the particle P-wave modulus. Here, we consider the packing behaviours beyond the jamming point as a continuum porous medium. $M^{\text {eff }}$ can hence be defined like the effective elastic P-wave modulus for a porous material:

$M^{\mathrm{eff}}=\frac{G^{\mathrm{eff}}\left(4 G^{\mathrm{eff}}-E^{\mathrm{eff}}\right)}{3 G^{\mathrm{eff}}-E^{\mathrm{eff}}}$.

Here, $E^{\text {eff }}$ is the effective Young's modulus given by [14]:

$E^{\mathrm{eff}}=E\left(1-\frac{p}{p_{c}}\right)^{f_{E}}$

and the effective shear modulus is [15]:
$G^{\mathrm{eff}}=G\left(1-\frac{p}{p_{c}}\right)^{f_{G}}$,

where $p$ is the porosity $(p=1-\Phi), f_{E}$ and $f_{G}$ denote the characteristic components for Young's and shear moduli, and $p_{c}$ is the critical porosity, below which the effective Young's and shear moduli become zero. This corresponds to below and near the jamming point.

The above relations are defined for the elastic particles. To consider the plastic deformation, the applied stress $\sigma$ may be decomposed into an elastic part $\sigma_{e}$ and a plastic one $\sigma_{p}$ as follows:

$\sigma=\left(1-f_{p}\right) \sigma_{e}+f_{p} \sigma_{p}$

where $f_{p}$ is the plastic volume fraction defined as the ratio between the volume of the plastic regions of particles and their total volume. During compaction process, $f_{p}$ is zero before the jamming, but beyond the jamming point, it is proportional to the vertical strain (hence, the packing fraction). One can therefore assume that $f_{p}$ increase logarithmically as a function of $\Phi / \Phi_{c}$ :

$f_{p}=\alpha \ln \left(\frac{\Phi}{\Phi_{c}}\right)$

where $\alpha$ is a constant parameter to be determined. Moreover, the plastic stress $\sigma_{p}$ can be defined in the following form by considering Eqs. (5) and (6):

$\begin{aligned} \sigma_{p} & =\sigma_{y}+M_{p}^{\mathrm{eff}}\left(\varepsilon-\varepsilon_{c}\right), \\ \sigma_{p} & =\sigma_{y}-\frac{M_{p}^{\mathrm{eff}}}{1+\frac{M_{p}^{\mathrm{eff}}}{c_{1_{p}} K_{p} Z \Phi}}\left(\ln (\Phi)+c_{2_{p}}\right),\end{aligned}$

with

$$
\begin{aligned}
& M_{p}^{\mathrm{eff}}=\frac{G_{p}^{\mathrm{eff}}\left(4 G_{p}^{\mathrm{eff}}-E_{p}^{\mathrm{eff}}\right)}{3 G_{p}^{\mathrm{eff}}-E_{p}^{\mathrm{eff}}} . \\
& E_{p}^{\mathrm{eff}}=E_{p}\left(1-\frac{p}{p_{c}}\right)^{f_{E_{p}}}, \\
& G_{p}^{\mathrm{eff}}=G_{p}\left(1-\frac{p}{p_{c}}\right)^{f_{G_{p}}} .
\end{aligned}
$$

Here, $E_{p}$ is the plastic tangent modulus related to $H$ through: $E_{p}=E H /(E+H)$ and $G_{p}$ denotes the plastic shear modulus: $G_{p}=E_{p} / 2\left(1+v_{p}\right)$. Note that, by assuming that the volume change due to plastic deformation is negligible, one concludes the equality of the elastic and plastic Poisson's ratios; i.e. $v_{p}=v$.

In order to determine several parameters in the elastic and plastic models (Eqs. (5), (6) and (10)), we consider first the elastic simulation results, allowing setting the elastic 


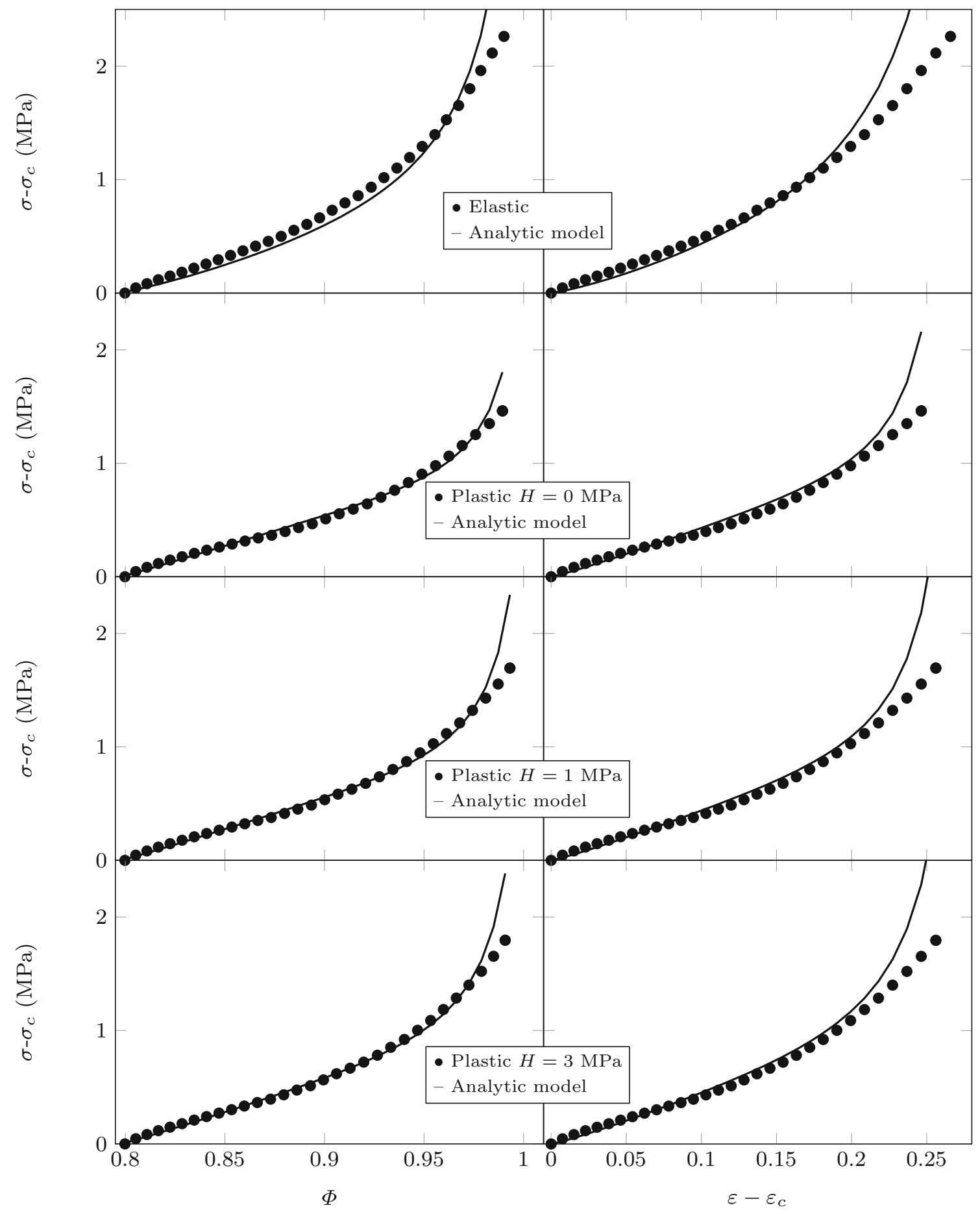

Fig. 4 Excess applied stress $\sigma-\sigma_{c}$ as a function of packing fraction $\Phi$ and excess strain $\varepsilon-\varepsilon_{c}$ for elastic and elasto-plastic (with three values of hardening, $H$ ) particles by MPM simulations. $\sigma_{c}$ and $\varepsilon_{c}$ correspond

parameters. In a similar vein, one can determine the plastic parameters from one of the plastic simulation results by fixing the obtained elastic parameters. In Fig. 4, we observe that the predictions of the elastic and plastic models are in good agree- to the jamming point $\Phi_{c} \simeq 0.8$. The lines show the predicted behaviours beyond jamming by the compaction models introduced in this paper; see Eqs. (5), (6) and (10)

ment with all MPM simulations by fixing $f_{E}=f_{E_{p}}=1.2$, $f_{G}=f_{G_{p}}=0.2$ and $p_{c}=0.201\left(\simeq 1-\Phi_{c}\right), c_{1}=c_{1_{p}}=1$, $c_{2}=c_{2_{p}}=0.23\left(\simeq-\ln \left(\Phi_{c}\right)\right)$ and $\alpha=2.5$. Nevertheless, at high packing fractions, a divergence between the models and 


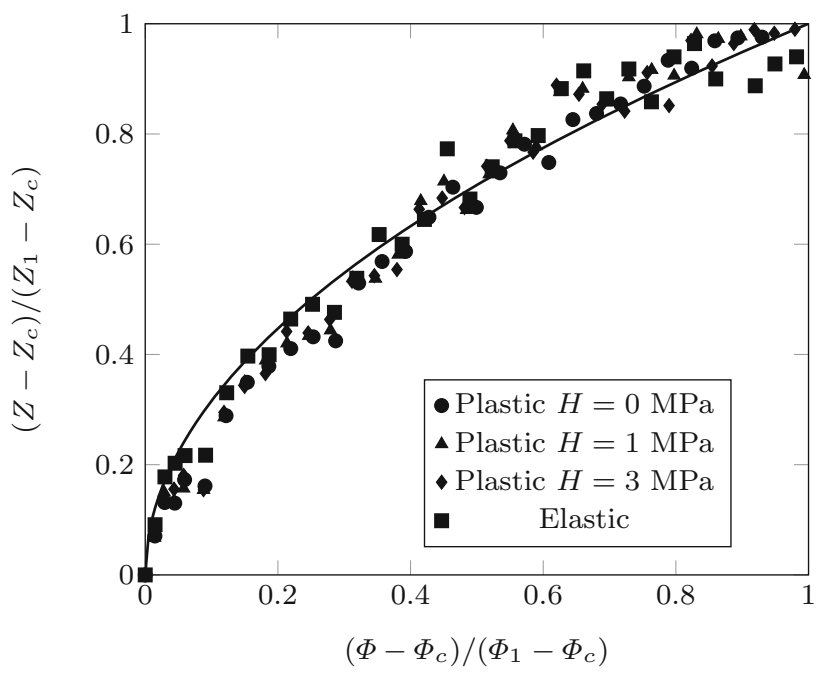

Fig. 5 Evolution of the excess mean coordination number $Z-Z_{c}$ as a function of excess packing fraction $\Phi-\Phi_{c}$ for elastic and elastoplastic particles by MPM simulations. The solid line is power-law fit $\left(\Phi-\Phi_{c}\right)^{0.5}$; see Eq. (17)

the numerical simulations is noticeable. To reproduce well the predictions, the particle discretisation may be refined in order to resolve correctly the small radii of curvature at the contact zones between particles. In consequence, to reproduce the compaction results, one needs to determine four parameters in the analytical models as well as the jamming state. Moreover, the general feature of the curves shown in Fig. 4 is more or less similar to the typical compaction curves observed in industrial applications [5,16-18] and the presented empirical model seems to reproduce well these results. However, this model still needs to be validated with a more detailed and quantitative comparison between simulations and experiments.

The evolution of the mean coordination number $Z$ beyond the jamming point as a function of the packing fraction is presented in Fig. 5. As shown in the previous works (e.g. [11, 19-21]), the excess coordination number $Z-Z_{c}$ normalised by $Z_{1}-Z_{c}$ behaves as a power law as a function of the excess packing fraction $\Phi-\Phi_{c}$ normalised by $\Phi_{1}-\Phi_{c}$ :

$$
\frac{Z-Z_{c}}{Z_{1}-Z_{c}}=\sqrt{\frac{\Phi-\Phi_{c}}{\Phi_{1}-\Phi_{c}}}
$$

where $Z_{1}$ and $\Phi_{1}$ correspond to any state after the jamming point. We observe that the elastic and plastic results are unified and fitted by the model (17) independent of the material behaviours.

\section{Concluding remarks}

This paper presents a compaction study of elasto-plastic soft particle systems using a numerical approach developed in our previous works [9-11]. This parallelised technique is based on coupling of an implicit formulation of the material point method (MPM) for individual particles and the contact dynamics (CD) method for the treatment of contact interactions. The MPM allows accounting for the realistic mechanical behaviour of individual particles, including elastic and plastic behaviours. Here, we consider the particle behaviour as a rate-independent elasto-plastic model based on the bilinear isotropic hardening. A diametrical compaction of one single particle was performed to illustrate the potential of this method to simulate contact interactions between soft elasto-plastic particles.

The MPM-CD approach is then used to simulate the uniaxial compaction of an assembly of soft particles (discs). Obviously, the discs do not represent real particle shapes in industrial applications. But the developed approach can be easily applied to more complex particle shapes [10]. The packings with plastic particles can undergo large deformations at a lower compressive stress in comparison with elastic particles. It is due to the occurrence of weaker stress chains between particles. However, the required compressive stress to achieve high packing fractions increases with hardening for plastic particles. We observed a logarithmic variation of this stress beyond the jamming state with packing fraction for elastic and plastic particles. A model based on porous materials was introduced in order to explain this behaviour. This model reproduced well the numerical results with four parameters to fix. Since the considered particles were nearly incompressible $(v=0.45)$, it is interesting to verify and adjust this model for different ranges of compressible particles. An experimental study is also required to validate the proposed model. Finally, it was shown that the coordination number is related to the packing fraction by a power-law function beyond the jamming transition.

Acknowledgements This work (Project ID 1502-607) was publicly funded through ANR (the French National Research Agency) under the "Investissements d'avenir" programme with the reference ANR-10LABX- 001-01 Labex Agro and coordinated by Agropolis Fondation, France, under the frame of I-SITE MUSE (ANR-16-IDEX-0006). We are also grateful to the Genotoul bioinformatics platform Toulouse Midi- Pyrenees (Bioinfo Genotoul) for providing computing resources.

\section{Compliance with ethical standards}

Conflict of interest The authors declare that they have no conflict of interest. 


\section{Appendix 1: Relation between the applied stress, $\sigma$, and the packing fraction, $\Phi$, for an elastic packing under uniaxial compression}

We assume that the packing of particles behaves almost as a continuum porous medium beyond the jamming point under uniaxial compression. Hence, in this range the applied stress $\sigma$ may be related to the cumulative vertical strain $\varepsilon$ through an effective $P$-wave modulus $M^{\text {eff }}$.

$\sigma=M^{\mathrm{eff}} \varepsilon$

One may further assume that the particle bulk modulus $K$ relates the volume increment $\mathrm{d} V_{S}$ of particles to the effective stress increment $\mathrm{d} \sigma_{S}$ in particles:

$K \frac{\mathrm{d} V_{S}}{V_{S}}=-\mathrm{d} \sigma_{S}$

$\sigma$ can be related to $\sigma_{S}$ as follows:

$\sigma=c_{1} Z \Phi \sigma_{S}$

where $c_{1}$ is a material constant to be determined. Given that $\mathrm{d} \varepsilon=\mathrm{d} V_{S} / V_{S}-\mathrm{d} \Phi / \Phi$ and using Eqs. (18), (19) and (20), the following differential equation to solve is obtained:

$$
\begin{aligned}
& \left(1+\frac{M^{\text {eff }}}{c_{1} K Z \Phi}\right) \mathrm{d} \sigma=\left[\frac{M^{\text {eff }}}{c_{1} K Z \Phi}\left(\frac{\mathrm{d} Z}{Z}+\frac{\mathrm{d} \Phi}{\Phi}\right)+\frac{\mathrm{d} M^{\text {eff }}}{M^{\text {eff }}}\right] \sigma \\
& -M^{\text {eff }} \frac{\mathrm{d} \Phi}{\Phi}
\end{aligned}
$$

By knowing that $M^{\text {eff }}$ and $Z$ are related to $\Phi$ (see Eqs. (7) and (17)), the integration of the differential equation (21) yields:

$\sigma=-\frac{M^{\mathrm{eff}}}{1+\frac{M^{\mathrm{eff}}}{c_{1} K Z \Phi}}\left(\ln (\Phi)+c_{2}\right)$,

where $c_{2}$ is the integral constant.

\section{References}

1. Sinka C (2007) Modelling powder compaction. KONA Powder Particle J 25:4

2. Pitt KG, Webber RJ, Hill KA, Dey D, Gamlen MJ (2015) Compression prediction accuracy from small scale compaction studies to production presses. Powder Technol 270:490
3. Wu CY, Ruddy O, Bentham A, Hancock B, Best S, Elliott J (2005) Modelling the mechanical behaviour of pharmaceutical powders during compaction. Powder Technol 152:107

4. Krok A, Peciar M, Fekete R (2014) Numerical investigation into the influence of the punch shape on the mechanical behavior of pharmaceutical powders during compaction. Particuology 16:116

5. Moghaddam M, Darvizeh R, Davey K, Darvizeh A (2018) Scaling of the powder compaction process. Int J Solids Struct 144:192

6. Wu CY (2008) DEM simulations of die filling during pharmaceutical tabletting. Particuology 6:412

7. Barnabe M, Blanc N, Chabin T, Delenne JY, Duri A, Frank X, Hugouvieux V, Lutton E, Mabille F, Nezamabadi S et al (2017) Multiscale modeling for bioresources and bioproducts. Innov Food Scie Emerg Technol 46: 41-53

8. Choi J, Gethin D (2009) A discrete finite element modelling and measurements for powder compaction. Model Simul Mater Sci Eng 17:035005

9. Nezamabadi S, Nguyen T, Delenne JY, Radjai F (2017) Modeling soft granular materials. Granul Matter 19:8

10. Nezamabadi S, Radjai F, Averseng J, Delenne JY (2015) Implicit frictional-contact model for soft particle systems. J Mech Phys Solids 83:72

11. Nezamabadi S, Frank X, Delenne JY, Averseng J, Radjai F (2019) Parallel implicit contact algorithm for soft particle systems. Comput Phys Commun 237:17

12. ANSYS (2009) ANSYS theory reference for the mechanical APDL and mechanical applications, 12th edn. In: ANSYS theory reference for the mechanical APDL and mechanical applications. ANSYS Inc., Canonsburg, PA

13. Andersen S, Andersen L (2010) Analysis of spatial interpolation in the material-point method. Comput Struct 88:506

14. Kováčik J (1999) Correlation between Young's modulus and porosity in porous materials. J Mater Sci Lett 8:1007

15. Kováčik J (2001) Correlation between shear modulus and porosity in porous materials. J Mater Sci Lett 20:1953

16. Samimi A, Hassanpour A, Ghadiri M (2005) Single and bulk compressions of soft granules: experimental study and DEM evaluation. Chem Eng Sci 60:3993

17. Stasiak M, Tomas J, Molenda M, Rusinek R, Mueller P (2010) Uniaxial compaction behaviour and elasticity of cohesive powders. Powder Technol 203:482

18. Zhou M, Huang S, Hu J, Lei Y, Zou F, Yan S, Yang M (2017) Experiment and finite element analysis of compaction densification mechanism of $\mathrm{Ag}-\mathrm{Cu}-\mathrm{Sn}-\mathrm{In}$ mixed metal powder. Powder Technol 313:68

19. O'Hern C, Silbert L, Liu A, Nagel S (2003) Jamming at zero temperature and zero applied stress: the epitome of disorder. Phys Rev E 68:011306

20. van Hecke M (2010) Jamming of soft particles: geometry, mechanics, scaling and isostaticity. J Phys Condens Matter 22:033101

21. Zhang J, Majmudar TS, Sperl M, Behringer R (2010) Jamming for a 2D granular material. Soft Matter 6:2982 